\title{
Developmental Dysplasia of the Hip: Management of Graf IIa Dysplastic Hip
}

\author{
Ali Ahmed Raba ${ }^{1,2,3 *}$, Qais Gasibat ${ }^{4,5,6}$ \\ ${ }^{1}$ UCD School of Medicine and Medical Sciences, Dublin, Ireland \\ ${ }^{2}$ Neonatology Department, Coombe Women and Infants University Hospital, Dublin, Ireland \\ ${ }^{3}$ School of Medicine, Misurata University, Misurata, Libya \\ ${ }^{4}$ Department of Sport Studies, Faculty of Educational Studies, Universiti Putra Malaysia (UPM), Kuala Lumpur, Malaysia \\ ${ }^{5}$ Department of Physiotherapy, Faculty of Medicine, Universiti Sultan Zainal Abidin, (UniSZA), Kuala Terengganu, Malaysia \\ ${ }^{6}$ Department of Physiotherapy, Faculty of Medical Technology, National Board for Technical and Vocational Education, Misurata, \\ Libya \\ Email: *ali.raba@ucdconnect.ie
}

How to cite this paper: Raba, A.A. and Gasibat, Q. (2020) Developmental Dysplasia of the Hip: Management of Graf IIa Dysplastic Hip. Open Journal of Pediatrics, $10,247-254$.

https://doi.org/10.4236/ojped.2020.102025

Received: June 28, 2019

Accepted: March 23, 2020

Published: April 26, 2020

Copyright $\odot 2020$ by author(s) and Scientific Research Publishing Inc. This work is licensed under the Creative Commons Attribution International License (CC BY 4.0).

http://creativecommons.org/licenses/by/4.0/

\begin{abstract}
Developmental Dysplasia of the Hip (DDH) describes a wide range of anatomic abnormalities of the growing hips which might result in permanent abnormal gait. Therefore, early detection and management is crucial to improve the outcome and minimise the risk of osteoarthritis in later life. Hip ultrasound is used to evaluate DDH based on Graf classification. Graf score is a widely used ultrasound grading score to evaluate DDH in infants. There is conflicting evidence regarding the management of the developmentally immature Graf type IIa hip. Some physicians adopt watchful waiting for natural maturation of hips while other early treatment. This article will provide an evidence-based review on the management modalities of the Graf type IIa hip.
\end{abstract}

\section{Keywords}

Developmental Dysplasia of the Hip, DDH, Graf IIa

\section{Introduction}

Developmental Dysplasia of the Hip (DDH) is a common musculoskeletal problem affecting newborn infants. DDH includes dislocation of femoral head from the acetabulum, subluxation of the femoral head and dysplasia of femoral head and acetabulum [1]. The incidence rates of DDH vary from $0.5 \%$ to $25 \%$ based on the geographical and ethnical origin [2]. DDH has been associated with multiple risk factors such as breech presentation, female sex, positive family history, 
first born baby, oligohydramnios and associated postural deformities such as torticollis and club foot [3]. Studies have shown that the risk of developing DDH is two-times higher in infants with at least one risk factor, compared with infants with no risk factors [4]. Genetically inherited abnormal hip development and/or joint laxity has commonly been considered as one of the main causes of DDH. It has been reported that there is a 12 -times increased risk for developing DDH if a newborn has a first-degree relative with DDH [5]. In addition to that, infants with positive family history of DDH have been found to have a slightly higher rate of failure in brace treatment than those without family history of DDH [5].

Early diagnosis and management is important to avoid an unfavourable long-term outcome. However, various studies revealed that surgery is not completely avoidable even if DDH was diagnosed and treated early. Ortolani and Barlow tests are most common screening tests which are commonly performed as a part of clinical examination of newborn infants [6]. These two tests are not useful in infants older than $6-8$ weeks as muscle strength increases, and capsule laxity reduces [7]. Dysplasia of the acetabulum can be missed by clinical examinations and can be diagnosed only by imaging [8].

Hips ultrasound is the investigation of choice within the first 3 months of age, and it is highly recommended for infants with risk factors for DDH or abnormal clinical examinations of the hips [9]. Various studies confirmed the high effectiveness of ultrasound scanning to evaluate $\mathrm{DDH}$, especially when compared with plain radiographs [10]. However, according to the American Academy of Pediatrics (AAP) guidelines, hip ultrasound and radiograph are equally effective imaging modalities for detecting DDH in infants with age of 4 - 6 months [8]. By 4 months of age, hip radiographs become more reliable, particularly when the ossification centre develops in the femoral head [8].

\section{Ultrasound Screening for DDH}

Ultrasound imaging allows the visualisation of the femoral head location relative to the acetabulum, and specific anatomic parameters, such as the depth of the acetabulum and inclination of the acetabular roof [11]. Ultrasound provides detailed visualisation of the cartilaginous anatomy of the hip that is not revealed by plain X-rays and allows early detection of DDH. Evidence is conflicting regarding the usage of ultrasound as standard screening test for DDH [12]. It has been reported that since the introduction of clinical and ultrasound screening of hips, the incidence of DDH has increased, which can be explained by the possibility of overdiagnosis of DDH [13]. In a Cochrane review on the effectiveness of different screening programs for DDH in newborn infants, it was found that the universal ultrasound screening is associated with an increased treatment rate with no significant change in the rate of late detected dysplasia or surgery [14]. Moreover, they revealed that targeted hips ultrasound to newborns at high risk of DDH did not significantly increase the treatment rate but also did not significantly reduce the rate of late detected dysplasia or surgery. 
Timing of ultrasound screening as part of the neonatal screening program for $\mathrm{DDH}$ remains an ongoing topic of debate. It is said that early ultrasound screenings can lead to a high incidence of false-positive or false-negative results, resulting in unnecessary anxiety, follow-up examinations, and financial burden [13] [14]. Universal ultrasound screening would cause an increased burden for studying a condition that affects 1 per 1000 newborns [15]. Studies have been conducted to identify the earliest possible time that ultrasound screening can be performed reliably for DDH. Gokharman et al. [16] chose the fourth and eighth weeks of life as the timepoints for their study because of the observation from their clinical practice that most of ultrasound examinations performed during the first 4 - 8 weeks of life. While, Lussier et al. [11] chose the threshold of 28 days for their study because most screenings at their institutions were performed either in the first week of life or after 28 days of life. Both studies were then unable to give a precise time point for the earliest timing that ultrasound screening could be reliably performed, since their comparison groups included patients from a wide range of time points before versus after the fourth week or 28 days of life. Controversy remains regarding which risk factors should be considered for performing an ultrasound screening. The European Society of Paediatrics Radiology considers breech presentation and positive family history as the only risk factors that indicate the necessity of performing a hip ultrasound when the neonatal physical examination is normal [17].

\section{Graf Classification}

Graf ultrasound has been used to evaluate DDH in children since the early 1980s [18]. Graf method is the most used in Europe and it allows for classification of the degree of severity of the anomalies of the hip. The infant remains in a lateral decubitus position and coronal images are taken with subsequent measurement of alpha and beta angles. The alpha angle refers to the angle between the acetabular roof and vertical cortex of the ilium. The beta angle is the angle formed between the vertical cortex of the ilium and the triangular labral fibrocartilage [19]. According to Graf Ultrasound, hips are classified to mature, immature, or dislocated. Type I hips are deemed mature, type III hips are referred to as immature (Table 1). In patients with early diagnosis, within 3 to 6 months of life, the treatment is usually conservative and involves the use of dynamic harness. The indication for the use of the Tubingen hip flexion splint is a dysplastic hip without instability, even if it could be utilised for hips types III and IV with a close sonographic surveillance, shifting to other different treatments if the results obtained, within 4 weeks, are not satisfactory [20].

\section{Graf IIa}

Hips with a slightly shallow acetabulum and rounded bony rim before 3 months of age are considered developmentally immature and are classified as Graf type IIa (Figure 1) [22]. The $\alpha$ angle is $50^{\circ}-59^{\circ}$, whereas the $\beta$ angle is $55^{\circ}-77^{\circ}$. These hips are clinically reduced and stable [23]. 
Table 1. Graf classification [21].

\begin{tabular}{|c|c|c|}
\hline Graf type & Angle & Incidence rate \\
\hline All type I & Alpha angle $>60$ degrees (normal) & \\
\hline Type IIa & Beta angle $<55$ degrees & $89 \%$ \\
\hline Type IIb & Beta angle $>55$ degrees & \\
\hline All type II & Alpha angle 50 - 59 degrees & $10.3 \%$ \\
\hline Type IIa & Alpha angle 50 - 59 degrees & \\
\hline Type IIb & Alpha angle 43 - 49 degrees & \\
\hline Type IIc & Beta angle $<77$ degrees & $0.2 \%$ \\
\hline Type D (“about to decenter") & $\begin{array}{l}\text { Alpha angle } 43-49 \text { degrees } \\
\text { Beta angle }>77 \text { degrees }\end{array}$ & $0.4 \%$ \\
\hline $\begin{array}{l}\text { Type III } \\
\text { Type IIIa and IIIb distinguished on } \\
\text { the grounds of structural alteration } \\
\text { of the cartilaginous roof }\end{array}$ & Alpha angle $<43$ degrees & $0.08 \%$ \\
\hline $\begin{array}{l}\text { Type IV (dislocated with labrum } \\
\text { interposed between femoral head } \\
\text { and acetabulum) }\end{array}$ & Alpha angle $<43$ degrees & $0.02 \%$ \\
\hline
\end{tabular}

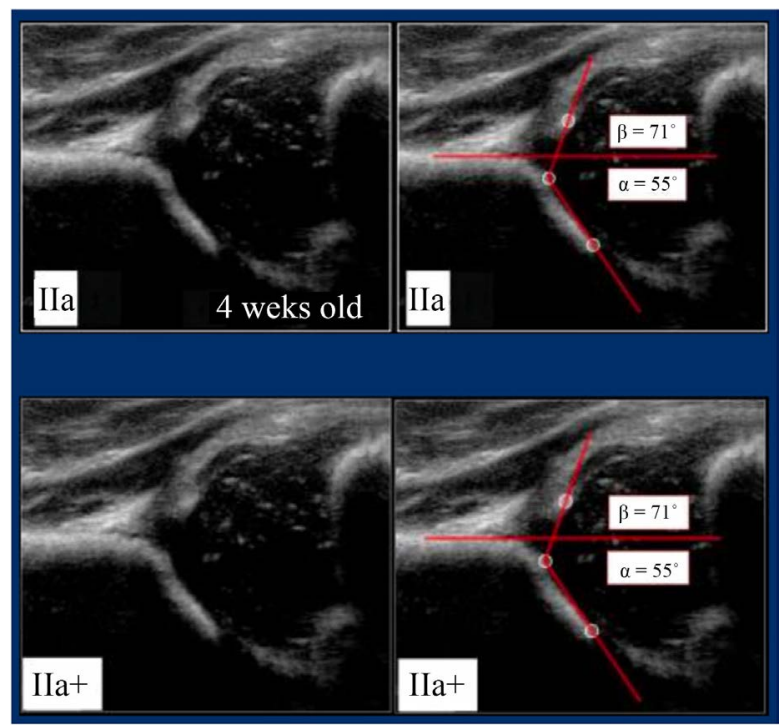

Figure 1. Graf Ultrasound showing Graf IIa DDH [21].

The prevalence of type IIa hips ranges from $10 \%$ to $45 \%$ depending on the age of the population [22]. The rate of spontaneous normalization in type IIa hips is reported to be $90 \%$ - 97\%, whereas dysplasia persists or worsens in $3 \%-10 \%$ of cases [22] [23]. Type IIa hips have been divided into two subtypes to differentiate hips that tend to deteriorate; type $\mathrm{IIa}(+)$ (physiologically immature) and type $\mathrm{IIa}(-)$ (maturational deficit), which should be decided according to the age in weeks and the $\alpha$ angle. Type IIa(+) hips are still within the acceptable limits for age. If a type IIa hip does not reach the minimum linear maturation rate, then it is called a type $\mathrm{IIa}(-)$ hip. The rate of missing the compulsory follow-up in type IIa hip is about one-third in the present series; this ratio is remarkably high. Very importantly, Graf type IIa hip should be carefully handled in newborn 
girls, as its natural history is not as good in girls as in boys [23].

\section{Management of Graf IIa}

The management of the infants with Graf Type IIa dysplastic hip is controversial. Some advocate early treatment with a harness whilst others adopt watchful waiting. The incidence of instability changes of infants with Graf IIa hip depending on the timing of the ultrasound examination because it commonly resolves as the infant gets older. The spontaneous normalization rate of type $\mathrm{IIa}(+)$ hip until the age of 3 months is about 95\% [24]. Management of type IIa(-) hips remains controversial and these hips always carry the risk of either overtreatment or development of true hip dysplasia due to delay in the required treatment [22]. About $85 \%$ of type IIa(-) hips reach normality without any treatment at the end of 3 months [24]. Therefore, Graf recommends treating the type $\mathrm{IIa}(-)$ hip by an abduction orthosis in order not to miss the valuable time window for normal acetabular development [24].

We have been following Graf's suggestion concerning the routine treatment of the type IIa(-) hip for many years. In the present series, among the completely followed type IIa hips, $15.6 \%$ became type $\mathrm{IIa}(-)$, so we began immediate treatment using Pavlik's method at the age of 6 - 7 weeks. It is obvious that prospective controlled studies comparing the effects of wait-and-see regimen and abduction orthosis treatment on the long-term outcome of the type IIa(-) hip are needed [20]. There is always the risk of missing physiologically immature and pathologic hips (Graf types IIa and IIb) by physical examination [24]. It has been observed that, after being informed about the benign nature of type IIa hips in most of the newborns, the parents commonly believe that their baby's minor hip problem will be completely resolved without any additional tests or treatments.

\section{The Effect of Risk Factors on the Natural Course of Graf IIa}

The correlation between DDH and different risk factors has been assessed, and inconsistent conclusions have been reported for many years. Although, the influence of various causative factors on the occurrence of DDH has widely been accepted, it is still not possible to put precisely one or more of these factors forward as the main cause(s) of DDH due to heterogeneity of the patient data and methodologies in different studies. Besides, to our knowledge, the correlation between the risk factors and the ultrasonographic types of hip pathology in patients [4]. Therefore, identifying the newborn infants with a higher risk of persistence or worsening of dysplasia is an important issue. Kosar et al. [22] reported that $5.6 \%$ of type IIa hips worsen sonographically at follow-up. Central nervous system anomalies, instability, and unilateral type IIa hips were predictors of worsening in their series [4]. This transition was more likely to occur in type IIa $(-)$ patients according to the initial $\alpha$ angle measurements. Also, girls are said to have a higher treatment rate than boys in type IIa hips of the present study. This is an expected finding as girls have a $5-8$ times higher incidence of 
DDH than boys. In addition, acetabular development in girls may be more deficient than that of boys during infancy.

Based on the findings, we can suggest that the type IIa hip should be carefully followed, especially in newborn girls; in the existence of a type $\mathrm{IIa}(-)$ hip in a girl, immediate treatment is always safer than a wait-and-see regimen to lessen the rate of possible complications including residual hip dysplasia and AVN [4] [20]. Both Pavlik harness and double nappies are safe treatment modalities for Type IIa hip dysplasia. However, sonographic deterioration was observed in both groups with surgical intervention required in the minority, supporting the ongoing treatment of these immature hips.

\section{Conclusion}

The incidence of type IIa hips ranges from 5\% - 12\% according to the time of the ultrasound examination. It has been reported that most cases with IIa hips spontaneously resolve when they get older. However, it is difficult to ascertain that all infants with Graf type IIa hips should be observed and evaluated for the natural course of type IIa hips using Graf perspective of physiological immaturity. Furthermore, ultrasound follow-up is necessary for all Graf type IIa hips; however, treatment is not necessary for all Graf type IIa hips. An $\alpha$ angle $<55^{\circ}$ on the initial ultrasound was an independent predictor for worsening, and this cut-off value should be considered to prevent future dysplasia. These patients usually require treatment with an orthosis, along with a more careful follow-up. Also, we see from various studies that type IIa hips were more common in newborn girls than in boys. Newborn boys' hips had a higher rate of spontaneous normalization than girls' hips at 6 - 7 weeks of age. More studies required to assess the natural course of the type IIa hips and to evaluate the effect of the risk factors for DDH on that course.

\section{Disclosure Statement}

This research did not receive any specific grant from funding agencies in the public, commercial, or not-for-profit sectors.

\section{Conflicts of Interest}

The authors declare no conflicts of interest regarding the publication of this paper.

\section{References}

[1] Guille, J.T., Pizzutillo, P.D. and MacEwen, G.D. (2000) Development Dysplasia of the Hip from Birth to Six Months. The Journal of the American Academy of Orthopaedic Surgeons, 8, 232-242. https://doi.org/10.5435/00124635-200007000-00004

[2] Puhan, M.A., Woolacott, N., Kleijnen, J. and Steurer, J. (2003) Observational Studies on Ultrasound Screening for Developmental Dysplasia of the Hip in Newborns: A Systematic Review. Ultraschall in der Medizin (Stuttgart, Germany: 1980), 24, 377-382. https://doi.org/10.1055/s-2003-45213 
[3] Abu Hassan, F.O. and Shannak, A. (2007) Associated Risk Factors in Children Who Had Late Presentation of Developmental Dysplasia of the Hip. Journal of Children's Orthopaedics, 1, 205-210. https://doi.org/10.1007/s11832-007-0041-5

[4] Ömeroğlu, H., Akceylan, A. and Köse, N. (2019) Associations between Risk Factors and Developmental Dysplasia of the Hip and Ultrasonographic Hip Type: A Retrospective Case Control Study. Journal of Children's Orthopaedics, 13, 161-166. https://doi.org/10.1302/1863-2548.13.180174

[5] Omeroğlu, H., Caylak, R., Inan, U. and Köse, N. (2013) Ultrasonographic Graf Type IIa Hip Needs More Consideration in Newborn Girls. Journal of Children's Orthopaedics, 7, 95-98. https://doi.org/10.1007/s11832-012-0476-1

[6] Agarwal, A. and Gupta, N. (2012) Risk Factors and Diagnosis of Developmental Dysplasia of Hip in Children. Journal of Clinical Orthopaedics and Trauma, 3, 10-14. https://doi.org/10.1016/j.jcot.2011.11.001

[7] Morey, S.S. (2001) AAP Develops Guidelines for Early Detection of Dislocated Hips. American Family Physician, 63, 565.

[8] (2000) Clinical Practice Guideline: Early Detection of Developmental Dysplasia of the Hip. Committee on Quality Improvement, Subcommittee on Developmental Dysplasia of the Hip. American Academy of Pediatrics. Pediatrics, 105, 896-905. https://doi.org/10.1542/peds.105.4.896

[9] Kotlarsky, P., Haber, R., Bialik, V. and Eidelman, M. (2015) Developmental Dysplasia of the Hip: What Has Changed in the Last 20 Years? World Journal of Orthopedics, 6, 886-901. https://doi.org/10.5312/wjo.v6.i11.886

[10] Bialik, V., Reuveni, A., Pery, M. and Fishman, J. (1989) Ultrasonography in Developmental Displacement of the Hip: A Critical Analysis of Our Results. Journal of Pediatric Orthopaedics, 9, 154-156. https://doi.org/10.1097/01202412-198909020-00006

[11] Lussier, E.C., Sun, Y.T., Chen, H.W., Chang, T.Y. and Chang, C.H. (2019) Ultrasound Screening for Developmental Dysplasia of the Hip after 4 Weeks Increases Exam Accuracy and Decreases Follow-Up Visits. Pediatrics and Neonatology, 60, 270-277. https://doi.org/10.1016/j.pedneo.2018.07.008

[12] Theis, J.C. and Vane, A. (2003) The Role of Ultrasound in Developmental Dysplasia of the Hip. Indian Journal of Orthopaedics, 37, 215.

[13] Bialik, V., Bialik, G.M., Blazer, S., Sujov, P., Wiener, F. and Berant, M. (1999) Developmental Dysplasia of the Hip: A New Approach to Incidence. Pediatrics, 103, 93-99. https://doi.org/10.1542/peds.103.1.93

[14] Shorter, D., Hong, T. and Osborn, D.A. (2013) Cochrane Review: Screening Programmes for Developmental Dysplasia of the Hip in Newborn Infants. Evidence-Based Child Health: A Cochrane Review Journal, 8, 11-54. https://doi.org/10.1002/ebch.1891

[15] Vaquero-Picado, A., González-Morán, G., Garay, E.G. and Moraleda, L. (2019) Developmental Dysplasia of the Hip: Update of Management. EFORT Open Reviews, 4, 548-556. https://doi.org/10.1302/2058-5241.4.180019

[16] Gokharman, F.D., Aydin, S., Fatihoglu, E., Ergun, E. and Kosar, P.N. (2019) Optimizing the Time for Developmental Dysplasia of the Hip Screening: Earlier or Later? Ultrasound Quarterly, 35, 130-135. https://doi.org/10.1097/RUQ.0000000000000348

[17] Tan, S.H.S., Wong, K.L., Lim, A.K.S. and Hui, J.H. (2019) The Earliest Timing of Ultrasound in Screening for Developmental Dysplasia of the Hips. Ultrasonography, 38, 321-326. https://doi.org/10.14366/usg.18075 
[18] Graf, R. (1981) The Ultrasonic Image of the Acetabular Rim in Infants: An Experimental and Clinical Investigation. Archives of Orthopaedic and Trauma Surgery, 99, 35-41. https://doi.org/10.1007/BF00400907

[19] Gulati, V., Eseonu, K., Sayani, J., Ismail, N., Uzoigwe, C., Choudhury, M.Z., Gulati, P., Aqil, A. and Tibrewal, S. (2013) Developmental Dysplasia of the Hip in the Newborn: A Systematic Review. World Journal of Orthopedics, 4, 32-41. https://doi.org/10.5312/wjo.v4.i2.32

[20] Pavone, V., Testa, G., Riccioli, M., Evola, F., Avondo, S. and Sessa, G. (2014) Treatment of Developmental Dysplasia of Hip with Tubingen Hip Flexion Splint. Journal of Pediatric Orthopaedics, 35, 485-489. https://doi.org/10.1097/BPO.0000000000000313

[21] Alexiev, V.A., Harcke, H.T. and Kumar, S.J. (2006) Residual Dysplasia after Successful Pavlik Harness Treatment: Early Ultrasound Predictors. Journal of Pediatric Orthopaedics, 26, 16-23. https://doi.org/10.1097/01.bpo.0000187995.02140.c7

[22] Kosar, P., Ergun, E., Gökharman, F.D., Turgut, A.T. and Kosar, U. (2011) Follow-Up Sonographic Results for Graf Type 2A Hips: Association with Risk Factors for Developmental Dysplasia of the Hip and Instability. Journal of Ultrasound in Medicine, 30, 677-683. https://doi.org/10.7863/jum.2011.30.5.677

[23] Bilgili, F., Sağlam, Y., Göksan, S.B., Hürmeydan, Ö.M., Birişik, F. and Demirel, M. (2018) Treatment of Graf Type IIa Hip Dysplasia: A Cut-Off Value for Decision Making. Balkan Medical Journal, 35, 427-430. https://doi.org/10.4274/balkanmedj.2017.1150

[24] Graf, R. (2006) Hip Sonography: Diagnosis and Management of Infant Hip Dysplasia. Springer, Berlin.

\section{Abbreviation}

Developmental Dysplasia of the Hip (DDH)

American Academy of Pediatrics (AAP) 